Also available at http://amc-journal.eu ISSN 1855-3966 (printed edn.), ISSN 1855-3974 (electronic edn.)

ARS MATHEMATICA CONTEMPORANEA 9 (2015) 261-266

\title{
On mixed discriminants of positively definite matrix*
}

\author{
Chang-Jian Zhao ${ }^{\dagger}$ \\ Department of Mathematics, China Jiliang University, \\ Hangzhou 310018, P. R. China \\ Xiao-Yan Li \\ Department of Mathematics, Hunan Normal University, \\ Changsha 410000, P. R. China
}

Received 2 November 2012, accepted 21 February 2014, published online 11 January 2015

\begin{abstract}
In the paper, some new inequalities for the mixed discriminants of positively definite matrix are established, which are the matrix analogues of inequalities of the well-known mixed volumes function.
\end{abstract}

Keywords: Mixed discriminants, symmetric matrix, mixed volume, Aleksandrov's inequality.

Math. Subj. Class.: 15A09, 52A40

\section{Introduction}

Let $x_{1}, \ldots, x_{n}$ be a set of nonnegative quantities and let $E_{i}(x)$ be the $i$-th elementary symmetric function of an $n$-tuple $x=x\left(x_{1}, \ldots, x_{n}\right)$ of non-negative reals is defined by $E_{0}(x)=1$ and

$$
E_{i}(x)=\sum_{1<j_{1}<\cdots<j_{i} \leq n} x_{j_{1}} x_{j_{2}} \cdots x_{j_{i}}, 1 \leq i \leq n .
$$

An interesting inequality for the symmetric function was established ([1], also see [2], p. 33) as follows.

$$
\frac{E_{i}(x+y)}{E_{i-1}(x+y)} \geq \frac{E_{i}(x)}{E_{i-1}(x)}+\frac{E_{i}(y)}{E_{i-1}(y)} .
$$

\footnotetext{
* Research is supported by National Natural Science Foundation of China (11371334).

${ }^{\dagger}$ Author to whom correspondence should be addressed.

E-mail addresses: chjzhao@163.com chjzhao@aliyun.com chjzhao315@sohu.com (Chang-Jian Zhao), lixy-77@163.com (Xiao-Yan Li)
} 
A matrix analogue of (1.1) is the following result of Bergstrom [3].

Let $K$ and $L$ be positive definite matrix, and let $K_{i}$ and $L_{i}$ denote the sub-matrices obtained by deleting the $i$-th row and column. Then

$$
\frac{\operatorname{det}(K+L)}{\operatorname{det}\left(K_{i}+L_{i}\right)} \geq \frac{\operatorname{det}(K)}{\operatorname{det}\left(K_{i}\right)}+\frac{\operatorname{det}(L)}{\operatorname{det}\left(L_{i}\right)}
$$

An interesting proof is due to Bellman [4] (also see [2], p. 67). A generalization of (1.2) was established by Ky Fan [5] (also see [6-7]). Moreover, we assume all positive definite matrix are supposed to be symmetric in the article.

There is a remarkable similarity between inequalities for symmetric functions (or determinants of symmetric matrices) and inequalities for the mixed volumes of convex bodies. In 1991, V. Milman asked if there is version of (1.1) or (1.2) in the theory of mixed volumes and it was stated as the following open question (see [8]):

Question 1.1. For which values of $i$ is it true that for very pair of convex bodies $K$ and $L$ in $\mathbb{R}^{n}$,

$$
\frac{W_{i}(K+L)}{W_{i+1}(K+L)} \geq \frac{W_{i}(K)}{W_{i+1}(K)}+\frac{W_{i}(L)}{W_{i+1}(L)} ?
$$

The convex body is the compact and convex subsets with non-empty interiors in $\mathbb{R}^{n}$. $W_{i}(K)$ denotes the quermassintegral of convex body $K$ and $W_{i+1}(K)$ denotes the mixed volumes $V(\underbrace{K, \ldots, K}_{n-i-1}, \underbrace{B, \ldots, B}_{i+1})$. The sum + is the usual Minkowski vector sum and $B$ denotes the unit ball.

A theorem by Minkowski provides a fundamental relation between volume and operations of addition and multiplication of convex bodies by nonnegative reals: If $K_{1}, \ldots, K_{m}$ are convex bodies, $m \in \mathbb{N}$, then the volume of $t_{1} K_{1}+\cdots+t_{m} K_{m}$ is a homogeneous polynomial of degree $n$ in $t_{i}>0$ (see [14]). That is

$$
V\left(t_{1} K_{1}+\cdots+t_{m} K_{m}\right)=\sum_{1 \leq i_{1}, \ldots, i_{n} \leq m} V\left(K_{i_{1}}, \ldots, K_{i_{n}}\right) t_{i_{1}} \cdots t_{i_{n}},
$$

where the coefficients $V\left(K_{i_{1}}, \ldots, K_{i_{n}}\right)$ are chosen to be invariant under permutations of their arguments. The coefficient $V\left(K_{i_{1}}, \ldots, K_{i_{n}}\right)$ is called the mixed volume of the $n$-tupe $\left(K_{i_{1}}, \ldots, K_{i_{n}}\right)$. Steiner's formula is a special case of Minkowski's theorem; the volume of $K+t B, t \geq 0$, can be expanded as a polynomial in $t$ :

$$
V(K+t B)=\sum_{i=0}^{n}\left(\begin{array}{l}
n \\
i
\end{array}\right) W_{i}(K) t^{i}
$$

where $W_{i}(K):=V(\underbrace{K, \ldots, K}_{n-i}, \underbrace{B, \ldots, B}_{i})$ is the quermassintegral of convex body $K$.

A partial answer ( $L$ must be a ball) of (1.3) was established by Gianopoulos, Hartzoulaki and Paouris [9]).

If $K$ is a convex body and $D$ is a ball in $\mathbb{R}^{n}$, then for $i=0, \ldots, n-1$

$$
\frac{W_{i}(K+D)}{W_{i+1}(K+D)} \geq \frac{W_{i}(K)}{W_{i+1}(K)}+\frac{W_{i}(D)}{W_{i+1}(D)} .
$$


The answer to the above question is negative; it can be proved that (1.3) is true in full generality only when $i=n-1$ or $i=n-2$ (the details see [10]). Moreover, a dual inequality of (1.4) for the dual quermassintegral of star bodies was proved by $\mathrm{Li}$ and Leng [11].

In the paper, we establish some inequalities for mixed discriminants of positively definite matrix which are matrix analogues of some mixed volumes inequalities.

\section{Mixed discriminants and Aleksandrov's inequality}

Recall that for positive definite $n \times n$ matrices $K_{1}, \ldots, K_{N}$ and $\lambda_{1}, \ldots, \lambda_{N} \geq 0$, the determinant of the linear combination $\lambda_{1} K_{1}+\cdots+\lambda_{N} K_{N}$ is a homogeneous polynomial of degree $n$ in the $\lambda_{i}$ (see e.g. [12]),

$$
\operatorname{det}\left(\lambda_{1} K_{1}+\cdots+\lambda_{N} K_{N}\right)=\sum_{1 \leq i_{1}, \ldots \ldots, i_{n} \leq N} D\left(K_{i_{1}}, \ldots, K_{i_{n}}\right) \lambda_{i_{1}} \cdots \lambda_{i_{n}},
$$

where the coefficient $D\left(K_{i_{1}}, \ldots, K_{i_{n}}\right)$ are chosen to be invariant under permutations of their arguments. The coefficient $D\left(K_{i_{1}}, \ldots, K_{i_{n}}\right)$ is called the mixed discriminant of $K_{i_{1}}, \ldots, K_{i_{n}}$.

The mixed discriminant $D(K, \ldots, K, I, \ldots, I)$, with $n-k$ copies of $K$ and $k$ copies of the identity matrix, $I$, will be abbreviated by $D_{k}(K)$. From $(2.1)$, we have

$$
D_{i}(K+\lambda I)=\sum_{j=0}^{n-i}\left(\begin{array}{l}
n-i \\
j
\end{array}\right) \lambda^{j} D_{i+j}(K)
$$

Note that the elementary mixed discriminants $D_{0}(K), \ldots, D_{n}(K)$ are thus defined as the coefficients of the polynomial

$$
\operatorname{det}(K+\lambda I)=\sum_{i=0}^{n}\left(\begin{array}{l}
n \\
i
\end{array}\right) \lambda^{i} D_{i}(K)
$$

Obviously, $D_{0}(K)=\operatorname{det}(K)$ while $n D_{n-1}(K)$ is the trace of $K$.

The well-known Aleksandrov's inequality for mixed discriminants can state as follows (see [13], also see [14], p.383 or [15], p.35):

Lemma 2.1. If $K_{1}, K_{2}, \ldots, K_{n}$ are real symmetric positively definite $n \times n$ matrices, then

$$
D\left(K_{1}, K_{2}, K_{3}, \ldots, K_{n}\right)^{2} \geq D\left(K_{1}, K_{1}, K_{3}, \ldots, K_{n}\right) D\left(K_{2}, K_{2}, K_{3}, \ldots, K_{n}\right),
$$

with equality if and only if $K_{1}=\lambda K_{2}$ with positive number $\lambda$.

\section{Inequalities for mixed discriminants of positively definite matrix}

Theorem 3.1. Let $K$ be symmetric positively definite matrix and I stands for the identity matrix and $t \geq 0$. If $0 \leq i \leq n-1$ and $i \in \mathbb{N}$, then the function

$$
g(t)=\frac{D_{i}(K+t I)}{D_{i+1}(K+t I)}
$$

is an increasing and concave function on $[0,+\infty)$. 
Proof. If $f_{i}(s)=D_{i}(K+s I)$, then by the linearity of the mixed discriminant we see that

$$
\begin{aligned}
f_{i}(s+\varepsilon) & =\sum_{j=0}^{n-i}\left(\begin{array}{c}
n-i \\
j
\end{array}\right) \varepsilon^{j} D_{i+j}(K+s I) \\
& =f_{i}(s)+\varepsilon(n-i) f_{i+1}(s)+o\left(\varepsilon^{2}\right) .
\end{aligned}
$$

Hence

$$
\begin{aligned}
\frac{d f_{i}(s)}{d s} & =\lim _{\varepsilon \rightarrow 0} \frac{f(s+\varepsilon)-f(s)}{\varepsilon} \\
& =(n-i) f_{i+1}(s) .
\end{aligned}
$$

Similarly, we obtain

$$
\frac{d f_{i+1}(s)}{d s}=(n-i-1) f_{i+2}(s) .
$$

From (2.4), we obtain for all $0 \leq i<n$

$$
f_{i+1}^{2}(s)-f_{i}(s) f_{i+2}(s) \geq 0,
$$

with equality if and only if $K=\mu I$.

From (3.2), (3.3) and (3.4), we have

$$
\begin{aligned}
& \frac{d f_{i}(s)}{d s} f_{i+1}(s)-f(s) \frac{d f_{i+1}(s)}{d s} \\
& \quad=f_{i+1}^{2}(s)+(n-i-1)\left(f_{i+1}^{2}(s)-f_{i}(s) f_{i+2}(s)\right) \\
& \quad \geq f_{i+1}^{2}(s) .
\end{aligned}
$$

Therefore

$$
\begin{aligned}
\frac{d g(s)}{d s} & =\left(\frac{f_{i}(s)}{f_{i+1}(s)}\right)^{\prime} \\
& =\frac{f_{i}^{\prime}(s) f_{i+1}(s)-f(s) f_{i+1}^{\prime}(s)}{f_{i+1}^{2}(s)} \\
& =(n-i)-(n-i-1) \frac{f_{i}(s) f_{i+2}(s)}{f_{i+1}^{2}(s)} .
\end{aligned}
$$

Hence

$$
f(t)=\frac{D_{i}(K+t I)}{D_{i+1}(K+t I)}
$$

is an increasing and concave function on $[0,+\infty)$.

Theorem 3.2. Let $K$ be symmetric positively definite matrix and I stands for the identity matrix. If $0 \leq i<n$, then

$$
\begin{aligned}
& (n-i) D_{i+2}(K)\left(D_{i+1}(K)^{2}-D_{i}(K) D_{i+2}(K)\right) \\
& \quad \geq(n-i-2) D_{i}(K)\left(D_{i+2}(K)^{2}-D_{i+1}(K) D_{i+3}(K)\right) .
\end{aligned}
$$


Proof. Let $f_{i}(t)=D_{i}(K+t I)$ for $t \geq 0$ and $g(t)=\frac{f_{i}(t)}{f_{i+1}(t)}$, then

$$
\frac{d g(s)}{d s}=(n-i)-(n-i-1) \frac{f_{i}(t) f_{i+2}(t)}{f_{i+1}^{2}(t)} .
$$

By differentiating the both sides of (3.6) again, we have

$$
\begin{gathered}
\frac{d^{2} g(s)}{d s^{2}}=-(n-i-1) \\
\times \frac{(n-i) f_{i+2}(t) f_{i+1}^{2}(t)+(n-i-2) f_{i}(t) f_{i+1}(t) f_{i+3}(t)-2(n-i-1) f_{i}(t) f_{i+2}^{2}(t)}{f_{i+1}^{3}(t)} .
\end{gathered}
$$

From (3.8) and in view of $g(s)$ being a concave function, we obtain $(n-i) f_{i+2}(t) f_{i+1}^{2}(t)+(n-i-2) f_{i}(t) f_{i+1}(t) f_{i+3}(t)-2(n-i-1) f_{i}(t) f_{i+2}^{2}(t) \geq 0$, for $t \in(0,+\infty)$.

This can be equivalently written in the form

$$
(n-i) f_{i+2}(t)\left(f_{i+1}^{2}(t)-f_{i}(t) f_{i+2}(t)\right) \geq(n-i-2) f_{i}(t)\left(f_{i+2}^{2}(t)-f_{i+1}(t) f_{i+3}(t)\right) .
$$

Hence

$$
\begin{aligned}
& (n-i) D_{i+2}(K+t I)\left(D_{i+1}(K+t I)^{2}-D_{i}(K+t I) D_{i+2}(K+t I)\right) \\
& \quad \geq(n-i-2) D_{i}(K+t I)\left(D_{i+2}(K)^{2}-D_{i+1}(K+t I) D_{i+3}(K+t I)\right) .
\end{aligned}
$$

Notice that $f_{i}(t)$ is continuous function, letting $t \rightarrow 0^{+}$in (3.10), (3.10) reduces to the inequality in Theorem 3.2.

\section{References}

[1] A. D. Aleksandrov, Zur Theorie der gemischten Volumina von konvexen Körpen, Die gemischten Diskriminanten und die gemischten Volumina (in Russian), Mat. Sbornik 3 (1938), 227 252.

[2] E. F. Bechenbach, R. Bellman, Inequalities, first ed., Springer-Verlag, Berlin-Göttingen, Heidelberg, 1961.

[3] R. Bellman, Notes on matrix theory-IV: an inequality due to Bergstrom, Amer. Math. Monthly 62 (1955), 172-173.

[4] H. Bergstrom, A triangle inequality for matrices. Den Elfte Skandinaviski Matematikerkongress, Trondheim, first ed., 1949, Oslo: John Grundt Tanums Forlag, 1952.

[5] A. Dembo, T. M. Cover and J. A. Thomas, Information theoretic inequalities, IEEE Trans. Infor. Theory 37 (1991), 1501-1518.

[6] K. Fan, Some inequalities conerning positive-definite hermitian matrices, Proc. Cambridge Phil. Soc. 51 (1955), 414-421.

[7] K. Fan, Problem 4786, Amer. Math. Monthly 65 (1958), 289. 
[8] M. Fradelizi, A. Giannopoulos and M. Meyer, Some inequalities about mixed volumes, Israel J. Math. 135 (2003), 157-179.

[9] A. Giannopoulos, M. Hartzoulaki and G. Paouris, On a local version of the AleksandrovFenchel inequality for the quermassintegrtals of a convex body, Proc. Amer. Math. Soc. 130 (2002), 2403-2412.

[10] X. Y. Li and G. S. Leng, Some inequalities about dual mixed volumes of star bodies, Acta Math. Sci. 25 (2005), 505-510.

[11] E. Lutwak, D. Yang and G. Zhang, A new affine invariant for polytopes and Schneider's projection problem, Trans. Amer. Math. Soc. 353 (2001), 1767-1779.

[12] M. Marcus and M. Marcus, Inequalities for symmetric functions and Hermitian matrices, Canad J. Math. 8 (1956), 524-531.

[13] L. Mirsky, Maximum principles in matrix theory, Proc. Glasgow Math. Assoc. 4 (1958), 34-37.

[14] P. Pranayanuntana, Elliptic Brunn-Minkowski Theory, first ed., UMT Dissertations Publishing, 2003.

[15] R. Schneider, Convex Bodies: The Brunn-Minkowski Theory, first ed., Cambridge Univ. Press, 1993. 